\title{
Analytical and Phenomenological Studies of Rotating Turbulence*
}

\author{
Alex Mahalov ${ }^{1}$ and Ye Zhou ${ }^{2}$ \\ 1 Department of Mathematics, Arizona State University, Tempe, AZ 85287 \\ ${ }^{2}$ Institute for Computer Applications in Science and Engineering \\ NASA Langley Research Center, Hampton, VA 23681
}

\begin{abstract}
A framework, which combines mathematical analysis, closure theory, and phenomenological treatment, is developed to study the spectral transfer process and reduction of dimensionality in turbulent flows that are subject to rotation. First, we outline a mathematical procedure that is particularly appropriate for problems with two disparate time scales. The approach which is based on the Green's method leads to the Poincaré velocity variables and the Poincaré transformation when applied to rotating turbulence. The effects of the rotation are now reflected in the modifications to the convolution of nonlinear term. The Poincaré transformed equations are used to obtain a time-dependent analog of the Taylor-Proudman theorem valid in the asymptotic limit when the non-dimensional parameter $\mu \equiv \Omega t \rightarrow \infty(\Omega$ is the rotation rate and $t$ is the time). The 'split' of the energy transfer in both direct and inverse directions is established. Secondly, we apply the Eddy-Damped-Quasinormal-Markovian (EDQNM) closure to the Poincaré transformed Euler/Navier-Stokes equations. This closure leads to expressions for the spectral energy transfer. In particular, an unique triple velocity decorrelation time is derived with an explicit dependence on the rotation rate. This provides an important input for applying the phenomenological treatment of Zhou. In order to characterize the relative strength of rotation, another non-dimensional number, a spectral Rossby number, which is defined as the ratio of rotation and turbulence time scales, is introduced. Finally, the energy spectrum and the spectral eddy viscosity are deduced.
\end{abstract}

*This research was supported by the National Aeronautics and Space Administration under NASA Contract No. NAS1-19480 while the authors were in residence at the Institute for Computer Applications in Science and Engineering (ICASE), NASA Langley Research Center, Hampton, VA 23681-0001. 


\section{Introduction}

Turbulent flows subject to solid body rotation have many important applications in aerospace engineering and geophysics. Although all turbulence models rely on assumptions on energy transfer, there is a great deal of difficulties in studying the time evolution of kinetic energy and dissipation of rotating turbulent flows. Although it has been long recognized that rotation can significantly modify properties of fluid flows, rotation does not even enter the transport equations for kinetic energy. This 'indirect' but significant influence by rotation is the physical root of the difficulties. A turbulence model which can account for rotation adequately has not yet been developed.

Experimental studies ${ }^{1-4}$, direct numerical simulations (DNS) $)^{5-8}$, large-eddy simulations $(\mathrm{LES})^{5,9}$, and closure approximation ${ }^{10}$ have established that solid body rotation suppresses the nonlinear energy cascade from large to small scales. The effect of rotation is through phase scrambling. Zhou ${ }^{11}$ recently noted the strong similarity between magnetohydrodynamic (MHD) turbulence and initially isotropic turbulence subject to rotation. For MHD turbulence, Kraichnan ${ }^{12}$ pointed out that the propagation of the Alfvénic fluctuations disrupts the phase relation and thereby may be expected, on the average, to decrease energy transfer. Similarly, uniform rotation causes plane waves to propagate with phase speed

$2 \Omega k_{3} / k$. Here the rotation vector is considered to act along the vertical $\left(\frac{\mathbf{x}}{|\mathbf{x}|}\right)$. This evidence formed the basis for extending the phenomenology originally developed for MHD turbulence to rotating turbulence ${ }^{11}$.

In this paper we focus our attention on developing a better understanding of the energy transfer process in homogeneous, isotropic, incompressible turbulence subjected to a uniform background rotation. In particular, we address the problem of suppression of energy transfer and reduction of dimensionality due to rotation. We also deduce an energy spectrum and spectral eddy viscosity that have an explicit dependence on rotation rate. Our analysis is based on the fact that this is a two time scale problem. We first present a general mathematical framework based on the Green's method which is applicable when there are disparate time scales. The approach leads to the Poincaré transformation and the Poincaré velocity variables. The latter is accomplished with the time-dependent change of velocity 
variables

$$
\mathbf{U}(t, x)=\mathbf{L}(t) \mathbf{u}=\int G(\Omega t, x, y) \mathbf{u}(t, y) d y .
$$

Here $\mathbf{L}(t)$ is the linear propagator describing propagation of inertial waves in rotating fluids (Poincaré ${ }^{13}$ ) and $G(\Omega t, x, y)$ is the Green's tensor corresponding to the gyroscopic operator. The effects of the rotation are now reflected in modifications of the convolution in the nonlinear term.

The transformed equations are used to obtain a time-dependent analog of the TaylorProudman theorem valid in the asymptotic limit $\mu=\Omega t \rightarrow+\infty$. The subtlety of the unsteady situation lies in the fact that purely $2 \mathrm{D}$ turbulence is unstable to $3 \mathrm{D}$ perturbations and is, therefore, un-realizable. Bershadskii, et al. ${ }^{14}$ argued that the quasi-2D turbulence regime arises as a result of a spontaneous breaking of reflexional symmetry, which in turn is a consequence of the instability of $2 \mathrm{D}$ turbulence to $3 \mathrm{D}$ wave motions. The experiments of Hopfinger et $a .^{15}$ beautifully demonstrated that two-dimensional rotating fluid columns are capable of supporting wave motions. The major problem in obtaining a faithful description of quasi2D turbulence is to account for $3 \mathrm{D}$ effects. To this end, we deduce an analog of the Taylor-Proudman theorem for time-dependent flows. The operation of vertical averaging applied to the Poincaré transformed equations is crucial in our analysis. The main conclusion is that in the above limit the vertically averaged velocity $\overline{\mathbf{U}}$ satisfies the classical $2 \mathrm{D}-3 \mathrm{C}$ Euler/Navier-Stokes equations with initial data averaged in the $x_{3}$ direction. The difference $\mathbf{V}=\mathbf{U}-\overline{\mathbf{U}}$ is, however, not small. The three-dimensional $\left(x_{3}\right.$-dependent $)$ vector field $\mathbf{V}$ is a passive vector transported by $\overline{\mathbf{U}}$. It satisfies equations with coefficients depending on $\overline{\mathbf{U}}$. The 'split' of the energy transfer in both direct and inverse directions is established: the vertically averaged velocity $\overline{\mathbf{U}}$ transfers energy to large scales (inverse cascade) as $2 \mathrm{D}$ turbulence; and the passive vector $\mathbf{V}$ transfers energy directly from large to small scales.

An EDQNM analysis ${ }^{16,17}$ is then performed on the Poincaré transformed rotating NavierStokes equations. Unlike the previous EDQNM analysis (EDQNM1 and EDQNM2) of Cambon and Jacquin ${ }^{10}$ which was based on the original rotating Navier-Stokes equation, our analysis leads to a unique time scale for the decorrelation of triple velocity correlations. This provides an important input for applying the phenomenological treatment of Zhou ${ }^{11}$. 
In this analysis, $\tau_{3}$, the time scale for decay of triple correlations, which is responsible for inducing turbulent spectral transfer, may depend on any relevant turbulence parameters. The usual assumptions, energy conservation by the nonlinear interaction and a local cascade, imply that $\epsilon$ is independent of wavenumber $k$. Here $\epsilon$ is the energy flux in the inertial range which also equals the dissipation rate. Local cascade implies that $\epsilon$ is explicitly proportional to $\tau_{3}$ and depends on the wavenumber and on the power of the omni-directional energy spectrum (Kraichnan ${ }^{12}$ ). A simple dimensional analysis leads to

$$
\epsilon=A^{2} \tau_{3}(k) k^{4} E^{2}(k)
$$

where $A$ is a constant. The Kolmogorov ${ }^{18}$ spectrum is recovered when $\tau_{3}(k)$ is chosen as a nonlinear time scale, $\tau_{n l}(k)$. Using the triple decorrelation time scale derived from EDQNM $^{16,17}$, we deduce the energy spectra and spectral eddy viscosity at asymptotic limits. In order to characterize the relative strength of rotation, a spectral Rossby number, defined as the ratio of rotation and turbulence time scales, is introduced. For rotating turbulence in equilibrium, the Rossby number is the only relevant parameter controlling the effects of rotation on the flow. However, for the non-equilibrium situation, two non-dimensional parameters are required.

Our analysis suggests the following picture of the energy transfer process in the limit of $\Omega t \rightarrow \infty$ and small spectral Rossby number (strong rotation). There are inverse energy transfers by $\overline{\mathbf{U}}$ with $-5 / 3$ omni-directional energy spectrum. This is the same as twodimensional turbulence. At the same time, there is also a direct energy cascade governed by the equation for the passive vector $\mathbf{V}$ at $\Omega t \rightarrow \infty$ and $\Omega>>1$. The energy spectrum of the total velocity is ' $k^{-2}$, by Zhou' ${ }^{11}$.

This paper is organized as follows. In Section 2, a mathematical procedure based on Green's method is described. The Poincaré transformation which leads to the NavierStokes equations for the Poincaré velocity variable is developed. The analog of the TaylorProudman theorem for time-dependent flows is derived in Section 3 by vertical averaging of the Poincaré transformed equations. Analysis of the Poincaré transformed energy transfer equations is presented in Section 4. The energy spectrum and spectral eddy viscosity are deduced in Section 5. The possibility of extension of the Green's method to study the effects 
of solid body rotation on flows in complex geometries is indicated in Section 6 .

\section{Poincaré transformed Euler/Navier-Stokes equations for rotating fluids}

We consider flows uniformly rotating with a constant angular velocity about the $x_{3}$ axis. The Navier-Stokes equations referred to rotating coordinates

$$
\begin{array}{r}
\frac{\partial}{\partial t} \mathbf{U}-\nu \nabla^{2} \mathbf{U}+\mathbf{U} \cdot \nabla \mathbf{U}+2 \Omega \mathbf{J} \mathbf{U}=-\nabla p, \\
\nabla \cdot \mathbf{U}=0 .
\end{array}
$$

Here $\mathbf{U}=\left(U_{1}, U_{2}, U_{3}\right)$ is the velocity field, $\mathbf{J}$ is the rotation matrix, $2 \Omega \mathbf{J} \mathbf{U}$ is Coriolis force and $p$ is the modified pressure. We note that $\{\mathbf{J U}\}_{\alpha}=\left\{e_{3} \times \mathbf{U}\right\}_{\alpha}=\epsilon_{\alpha 3 j} U_{j}$ where $\epsilon_{i j l}$ is the antisymmetric tensor of order 3 , is not equal to zero only if $i j l$ are different, and equal to 1 if the permutation $i j l$ is even and -1 if the permutation is odd.

Eq. (2.1) can be written in the component form

$$
\begin{array}{r}
U_{\alpha, t}+U_{j} U_{\alpha, j}-\nu U_{\alpha, j j}+2 \Omega \epsilon_{\alpha 3 j} U_{j}=-p_{, \alpha} \\
U_{\alpha, \alpha}=0 .
\end{array}
$$

We introduce the Leray projection operator on the divergence free vector fields denoted by P. Applying $\mathbf{P}$ to Eq. (2.1), we obtain

$$
\frac{\partial}{\partial t} \mathbf{U}-\nu \nabla^{2} \mathbf{U}+\mathbf{P}\{\mathbf{U} \cdot \nabla \mathbf{U}\}+2 \Omega \mathbf{P J} \mathbf{U}=0 .
$$

We note that $\mathbf{P}\{\mathbf{U} \cdot \nabla \mathbf{U}\}=-\mathbf{P}\{\mathbf{U} \times$ curl $\mathbf{U}\}$. The pressure is eliminated in favor of the tensor $\mathbf{P}(\mathbf{k})=\left\{P_{\alpha \beta}(\mathbf{k})\right\}\left(P_{\alpha \beta}(\mathbf{k})=\delta_{\alpha \beta}-\frac{k_{\alpha} k_{\beta}}{k^{2}}, k=|\mathbf{k}|\right.$ in Fourier space $)$ using the incompressibility condition. Direct calculation shows that $\mathbf{P}(\mathbf{k}) \mathbf{J U}(\mathbf{k})=\mathbf{P}(\mathbf{k}) \mathbf{J P}(\mathbf{k}) \mathbf{U}(\mathbf{k})=\frac{k_{3}}{k^{2}} \epsilon_{\alpha l m} k_{l} U_{m}(\mathbf{k})$. Transforming to wavenumber space, the equation of motion (2.3) written in the component form reads

$$
\frac{\partial}{\partial t} U_{\alpha}(\mathbf{k}, t)+\nu k^{2} U_{\alpha}(\mathbf{k}, t)+2 \Omega \frac{k_{3}}{k^{2}} \epsilon_{\alpha l m} k_{l} U_{m}(\mathbf{k})=-\frac{i}{2} P_{\alpha l m}(\mathbf{k}) \sum_{\mathbf{p}+\mathbf{q}=\mathbf{k}} U_{l}(\mathbf{p}, t) U_{m}(\mathbf{q}, t)
$$


where

$$
P_{\alpha \beta \gamma}(\mathrm{k})=k_{\beta} P_{\alpha \gamma}(\mathrm{k})+k_{\gamma} P_{\alpha \beta}(\mathrm{k}) .
$$

For $\Omega=0$ we obtain equations which are the starting point of EDQNM analysis of nonrotating turbulence presented by $\operatorname{Orszag}^{16}$.

Next we introduce the Poincaré velocity $\mathbf{u}$ related to $\mathbf{U}$ as follows

$$
\mathbf{U}=\exp (-2 \Omega \mathbf{P J} t) \mathbf{u}=\int G(-\Omega t, x, y) \mathbf{u}(y) d y .
$$

Here $G$ is the Green's function of the gyroscopic operator (e.g. Ladyzhenskaya ${ }^{19}$ ). The identity Eq. (2.6) can be easily inverted: $\mathbf{u}=\exp (2 \Omega \mathbf{P J} t) \mathbf{U}=\int G(\Omega t, x, y) \mathbf{u}(y) d y$. We find that the Poincaré velocity $\mathbf{u}$ is solenoidal: $\nabla \cdot \mathbf{u}=0$. The operator $\exp (-2 \Omega \mathbf{P J} t)$ is the linear propagator studied by Poincaré ${ }^{13}$. The mathematical theory of the Poincaré propagator has attracted a considerable amount of attention since the work of Sobolev ${ }^{20}$. In particular, a number of results on the structure of this operator in bounded domains are available (Ladyzhenskaya ${ }^{19}$ ). They can be used to study the effects of background rotation on turbulence in complex geometries. In this paper we restrict our attention to homogeneous flows. Possible applications of the Green's function method in complex geometries will be indicated in Section 6.

Eq. (2.3) written in Poincaré $\mathbf{u}$-variables becomes

$$
\begin{array}{r}
\frac{\partial}{\partial t} \mathbf{u}-\nu \nabla^{2} \mathbf{u}=\exp (2 \Omega \mathbf{P J} t)\{\exp (-2 \Omega \mathbf{P J} t) \mathbf{u} \times \operatorname{curl}(\exp (-2 \Omega \mathbf{P J} t) \mathbf{u})\} \\
\nabla \cdot \mathbf{u}=0 .
\end{array}
$$

The Poincaré transformation (2.6) which relates velocity variables $\mathbf{U}$ and $\mathbf{u}$ can be written in the component form as follows

$$
U_{\alpha}(\mathrm{k}, t)=\cos \left(\frac{k_{3}}{k} 2 \Omega t\right) u_{\alpha}(\mathrm{k}, t)-\frac{1}{k} \sin \left(\frac{k_{3}}{k} 2 \Omega t\right) \epsilon_{\alpha l m} k_{l} u_{m}(\mathrm{k}, t) .
$$

This transformation eliminates rotational term in (2.4) but alters the nonlinear term as shown below.

It is convenient to introduce a $3 \times 3$ orthogonal matrix $M=\left\{M_{\alpha \gamma}\right\}$ defined on the linear subspace of vectors $\mathbf{u}(k)$ satisfying $\mathbf{k} \cdot \mathbf{u}(\mathbf{k})=0$ :

$$
M_{\alpha \gamma}(\mathrm{k}, \Omega t)=\cos \left(\frac{k_{3}}{k} 2 \Omega t\right) \delta_{\alpha \gamma}+\frac{1}{k} \sin \left(\frac{k_{3}}{k} 2 \Omega t\right) \epsilon_{\alpha l \gamma} k_{l} .
$$


The inverse of the $3 \times 3$ matrix $M$ denoted by $M^{-1}=\left\{\tilde{M}_{\alpha \gamma}\right\}$ is easily computed as

$$
\tilde{M}(\mathrm{k}, \Omega t) \equiv M^{-1}(\mathrm{k}, \Omega t)=M(\mathrm{k},-\Omega t) .
$$

Then the Poincaré transformation (2.8) is inverted as follows

$$
u_{\alpha}(\mathbf{k}, t)=\cos \left(\frac{k_{3}}{k} 2 \Omega t\right) U_{\alpha}(\mathbf{k}, t)+\frac{1}{k} \sin \left(\frac{k_{3}}{k} 2 \Omega t\right) \epsilon_{\alpha l m} k_{l} U_{m}(\mathbf{k}, t) .
$$

Using the relation (2.8), we obtain

$$
\begin{gathered}
\frac{\partial}{\partial t} U_{\alpha}(\mathrm{k}, t)+\nu k^{2} U_{\alpha}(\mathrm{k}, t)+2 \Omega \frac{k_{3}}{k^{2}} \epsilon_{\alpha l m} k_{l} U_{m}(\mathrm{k}, t)= \\
\cos \left(\frac{k_{3}}{k} 2 \Omega t\right) \frac{\partial u_{\alpha}}{\partial t}(\mathrm{k}, t)-\frac{1}{k} \sin \left(\frac{k_{3}}{k} 2 \Omega t\right) \epsilon_{\alpha l m} k_{l} \frac{\partial u_{m}}{\partial t}(\mathrm{k}, t) .
\end{gathered}
$$

Therefore,

$$
\begin{array}{r}
\cos \left(\frac{k_{3}}{k} 2 \Omega t\right) \frac{\partial u_{\alpha}}{\partial t}(\mathbf{k}, t)-\frac{1}{k} \sin \left(\frac{k_{3}}{k} 2 \Omega t\right) \epsilon_{\alpha l m} k_{l} \frac{\partial u_{m}}{\partial t}(\mathbf{k}, t)+\nu k^{2} M_{\alpha m}(\mathbf{k},-\Omega t) u_{m}(\mathbf{k}, t)= \\
-\frac{i}{2} P_{\alpha l m}(\mathbf{k}) \sum_{\mathbf{p}+\mathbf{q}=\mathbf{k}} U_{l}(\mathbf{p}, t) U_{m}(\mathbf{q}, t) .(2.13)
\end{array}
$$

Recall that the velocity vector $\mathbf{U}$ is expressed in terms of the Poincaré velocity $\mathbf{u}$ via the Poincaré transformation.

Eq. (2.13) can be written in the equivalent form

$$
M(\mathbf{k},-\Omega t)\left(\frac{\partial \mathbf{u}(\mathbf{k}, t)}{\partial t}+\nu k^{2} \mathbf{u}(\mathbf{k}, t)\right)=-\frac{i}{2} \sum_{\mathbf{p}+\mathbf{q}=\mathbf{k}} U_{l}(\mathbf{p}, t) U_{m}(\mathbf{q}, t)\left(\begin{array}{c}
P_{1 l m}(\mathbf{k}) \\
P_{2 l m}(\mathbf{k}) \\
P_{3 l m}(\mathbf{k})
\end{array}\right) .
$$

Inverting the $3 \times 3$ orthogonal matrix $M(\mathrm{k},-\Omega t)\left(M^{-1}(\mathrm{k},-\Omega t)=M(\mathrm{k}, \Omega t)\right.$ by $\left.(2.10)\right)$, we obtain

$$
\frac{\partial \mathbf{u}(\mathbf{k}, t)}{\partial t}+\nu k^{2} \mathbf{u}(\mathbf{k}, t)=-\frac{i}{2} \sum_{\mathbf{p}+\mathbf{q}=\mathbf{k}} U_{l}(\mathbf{p}, t) U_{m}(\mathbf{q}, t) M(\mathbf{k}, \Omega t)\left(\begin{array}{c}
P_{1 l m}(\mathbf{k}) \\
P_{2 l m}(\mathbf{k}) \\
P_{3 l m}(\mathbf{k})
\end{array}\right) .
$$

We rewrite $(2.15)$ in the component form 


$$
\begin{aligned}
& \frac{\partial}{\partial t} u_{\alpha}(\mathbf{k}, t)+\nu k^{2} u_{\alpha}(\mathbf{k}, t) \\
& =-\frac{i}{2}\left[\cos \left(\frac{k_{3}}{k} 2 \Omega t\right) P_{\alpha l m}(\mathbf{k})+\sin \left(\frac{k_{3}}{k} 2 \Omega t\right) \epsilon_{\alpha \xi \sigma} \frac{k_{\xi}}{k} P_{\sigma l m}(k)\right] \sum_{\mathbf{p}+\mathbf{q}=\mathbf{k}} U_{l}(\mathbf{p}, t) U_{m}(\mathbf{q}, t) \text {. }
\end{aligned}
$$

Substituting expressions for $U_{l}$ and $U_{m}$ in terms of $u$ via the Poincaré transformation (2.8), we obtain the Poincaré transformed Navier-Stokes equations written in terms of the Poincaré velocity $\mathbf{u}$ as follows:

$$
\frac{\partial}{\partial t} u_{\alpha}(\mathbf{k}, t)+\nu k^{2} u_{\alpha}(\mathbf{k}, t)=-\frac{i}{2} \sum_{\mathbf{p}+\mathbf{q}=\mathbf{k}} F_{\alpha \beta \gamma}(\mathbf{k}, \mathbf{p}, \mathbf{q}, 2 \Omega t) u_{\beta}(p, t) u_{\gamma}(q, t)
$$

where

$$
F_{\alpha \beta \gamma}(\mathbf{k}, \mathbf{p}, \mathbf{q}, 2 \Omega t)=\sum_{j=1}^{8} F_{\alpha \beta \gamma}^{j}(\mathbf{k}, \mathbf{p}, \mathbf{q}, 2 \Omega t)=\sum_{j=1}^{8} f^{j}(\mathbf{k}, \mathbf{p}, \mathbf{q}, 2 \Omega t) Q_{\alpha \beta \gamma}^{j}(\mathbf{k}, \mathbf{p}, \mathbf{q}) .
$$

Here

$$
\begin{aligned}
& Q_{\alpha \beta \gamma}^{1}(\mathbf{k}, \mathbf{p}, \mathbf{q})=P_{\alpha \beta \gamma}(\mathbf{k}), \\
& Q_{\alpha \beta \gamma}^{2}(\mathbf{k}, \mathbf{p}, \mathbf{q})=-P_{\alpha \beta m}(\mathbf{k}) \frac{1}{q} \epsilon_{m h \gamma} q_{h} \\
& Q_{\alpha \beta \gamma}^{3}(\mathbf{k}, \mathbf{p}, \mathbf{q})=-P_{\alpha l \gamma}(\mathbf{k}) \frac{1}{p} \epsilon_{l s \beta} p_{s} \\
& Q_{\alpha \beta \gamma}^{4}(\mathbf{k}, \mathbf{p}, \mathbf{q})=P_{\alpha l m}(\mathbf{k}) \frac{1}{p} \epsilon_{l s \beta} p_{s} \frac{1}{q} \epsilon_{m h \gamma} q_{h}, \\
& Q_{\alpha \beta \gamma}^{5}(\mathbf{k}, \mathbf{p}, \mathbf{q})=\epsilon_{\alpha \xi \sigma} \frac{k_{\xi}}{k} P_{\sigma \beta \gamma}(\mathbf{k}), \\
& Q_{\alpha \beta \gamma}^{6}(\mathbf{k}, \mathbf{p}, \mathbf{q})=-\epsilon_{\alpha \xi \sigma} \frac{k_{\xi}}{k} P_{\sigma \beta m}(\mathbf{k}) \epsilon_{m h \gamma} \frac{q_{h}}{q}, \\
& Q_{\alpha \beta \gamma}^{7}(\mathbf{k}, \mathbf{p}, \mathbf{q})=-\epsilon_{\alpha \xi \sigma} \frac{k_{\xi}}{k} P_{\sigma l \gamma}(\mathbf{k}) \epsilon_{l s \beta} \frac{p_{s}}{p}, \\
& Q_{\alpha \beta \gamma}^{8}(\mathbf{k}, \mathbf{p}, \mathbf{q})=\epsilon_{\alpha \xi \sigma} \frac{k_{\xi}}{k} P_{\sigma l m}(\mathbf{k}) \epsilon_{l s \beta} \frac{p_{s}}{p} \epsilon_{m h \gamma} \frac{q_{h}}{q}
\end{aligned}
$$

and

$$
f^{1}(\mathbf{k}, \mathbf{p}, \mathbf{q}, 2 \Omega t)=\operatorname{ccc}(k, p, q, 2 \Omega t)=\cos \left(\frac{k_{3}}{k} 2 \Omega t\right) \cos \left(\frac{p_{3}}{p} 2 \Omega t\right) \cos \left(\frac{q_{3}}{q} 2 \Omega t\right),
$$




$$
f^{8}(\mathbf{k}, \mathbf{p}, \mathbf{q}, 2 \Omega t)=\operatorname{sss}(k, p, q, 2 \Omega t)=\sin \left(\frac{k_{3}}{k} 2 \Omega t\right) \sin \left(\frac{p_{3}}{p} 2 \Omega t\right) \sin \left(\frac{q_{3}}{q} 2 \Omega t\right) .
$$

In the limit $\mu=\Omega t \rightarrow 0$, the first term, $j=1$, is the leading order term. The non-rotating limit can be recovered from the $f^{1}(\mathbf{k}, \mathbf{p}, \mathbf{q}, 2 \Omega t)$ term by setting $\Omega=0$ in Eq. (2.17):

$$
\frac{\partial}{\partial t} u_{\alpha}(\mathbf{k}, t)+\nu k^{2} u_{\alpha}(\mathbf{k}, t)=-\frac{i}{2} P_{\alpha \beta \gamma}(\mathbf{k}) \sum_{p+q=k} u_{\beta}(\mathbf{p}, t) u_{\gamma}(\mathbf{q}, t) .
$$

We note that the Coriolis term $2 \Omega \frac{k_{3}}{k^{2}} \epsilon_{\alpha l m} k_{l} U_{m}(\mathrm{k})$ in Eq. (2.4) (after projection on the divergence free subspace) has the form of a scalar times the curl operator. Therefore, eigenfunctions of this operator coincide with eigenfunctions of the curl operator. Decomposition of velocity fields along eigenfunctions of the curl operator is known as the "helical wave decomposition" and has attracted a considerable amount of attention since the work of Moffatt $^{21}$, Moses ${ }^{22}$, Lesieur ${ }^{17}$ and others. More recently, it was used in investigations of the energy transfer in both non-rotating and rotating turbulent flows ${ }^{10,23}$ and for weakly compressible turbulence ${ }^{24}$. The main focus of these analyses is amplitude equations describing helical wave interactions. In the present paper we prefer to work directly with velocity variables without reverting to the helical wave amplitude equations. Our EDQNM development, presented in detail in the Appendix, is a natural extension to turbulence subjected to a uniform background rotation of Orszag's ${ }^{16}$ EDQNM analysis of non-rotating turbulence.

\section{Dynamics of vertically averaged velocity and the anal- ogy of the Taylor-Proudman theorem for time-dependent flows}

In this section we use the Poincaré transformed equations to deduce an analog of the TaylorProudman theorem for time-dependent flows valid in the asymptotic limit $\mu=\Omega t \rightarrow+\infty$. The Taylor-Proudman theorem in its classical form states that steady inviscid flows in a rapidly rotating frame are two-dimensional, i.e. are independent of the coordinate along the axis of rotation of the fluid (Tritton ${ }^{25}$, Speziale ${ }^{26}$ ). The importance of this statement stems from its use in calibration of turbulence models for rotating flows. According to Speziale ${ }^{26}$, 
it is essential that turbulence models be developed whose properties in non-inertial frames of reference are consistent with the Taylor-Proudman theorem describing the structure of solutions of the Navier-Stokes equations in a rapidly rotating frame.

The subtlety of the unsteady situation is, in the fact, that purely two-dimensional turbulence is unstable to three-dimensional perturbations and, therefore, unrealizable. The fact that two-dimensional rotating fluid columns are capable of supporting $3 \mathrm{D}$ ( $x_{3}$-dependent) wave motions is beautifully demonstrated in the experiments of Hopfinger et al. ${ }^{15}$. The major problem is to account for the collective contribution to the dynamics made by wave motions. To this end, we deduce an analog of the Taylor-Proudman theorem for timedependent flows.

The main idea is to obtain equations for the vertically averaged velocity using the formulation of the problem in terms of Poincaré velocity variables. We find that the vertically averaged equations have a particularly simple form in the asymptotic limit $\Omega t \rightarrow+\infty$. The main part of the equations coincides with the two-dimensional, three components (2D3C) Euler/Navier-Stokes equations. The additional term involves terms varying only on a fast time scale. The structure of these equations allows us to obtain the analog of the Taylor-Proudman theorem for time-dependent flows.

In this section we use the notation $\bar{u}_{\alpha}$ for the vertical (the direction of rotation) average of $u_{\alpha}: \bar{u}_{\alpha}=\int u_{\alpha} d x_{3}$. Let $\mathbf{k}^{\prime}=\left(k_{1}, k_{2}, 0\right)$ where $\mathbf{k}^{\prime}$ is the $2 \mathrm{D}$ horizontal component of the wavevector k. Vertical averaging in Fourier space corresponds to restricting Fourier sums to wavevectors satisfying the condition $k_{3}=0$. Thus, $\overline{u_{\alpha}(\mathbf{k})}=u_{\alpha}\left(\mathbf{k}^{\prime}\right)$ is a $2 \mathrm{D}-3 \mathrm{C}$ velocity field. An important observation is that $\overline{\mathbf{U}}=\overline{\exp (-2 \Omega \mathrm{PJ} t) \mathbf{u}}=\overline{\mathbf{u}}$ since $\left.M(\mathrm{k}, \Omega t)\right|_{k_{3}=0}=I d(3 \times 3$ identity matrix) which follows from (2.9). Applying the operation of vertical averaging to $\mathrm{Eq} \cdot(2.16)$, we obtain

$$
\begin{array}{r}
\frac{\partial}{\partial t} u_{\alpha}\left(\mathbf{k}^{\prime}, t\right)+\nu k^{2} u_{\alpha}\left(\mathbf{k}^{\prime}, t\right)=-\frac{i}{2} \overline{P_{\alpha l m}(\mathbf{k}) \sum_{\mathbf{p}+\mathbf{q}=\mathbf{k}} U_{l}(\mathbf{p}, t) U_{m}(\mathbf{q}, t)}= \\
-\frac{i}{2} \sum_{\mathbf{p}+\mathbf{q}=\mathbf{k}, k_{3}=p_{3}+q_{3}=0} P_{\alpha l m}(\mathbf{k}) U_{l}(\mathbf{p}, t) U_{m}(\mathbf{q}, t) .
\end{array}
$$

Recall that the velocity vector $\mathbf{U}$ is related to the Poincaré velocity vector $\mathbf{u}$ by Eq. (2.8). 
Then Eq. (3.1) together with $k_{3}=0$ implies

$$
\frac{\partial}{\partial t} u_{\alpha}\left(\mathbf{k}^{\prime}, t\right)+\nu k^{2} u_{\alpha}\left(\mathbf{k}^{\prime}, t\right)=-\frac{i}{2} \sum_{\mathbf{p}^{\prime}+\mathbf{q}^{\prime}=\mathbf{k}^{\prime}, p_{3}+q_{3}=0} \hat{F}_{\alpha \beta \gamma}\left(\mathbf{k}^{\prime}, \mathbf{p}, \mathbf{q}, 2 \Omega t\right) u_{\beta}(\mathbf{p}, t) u_{\gamma}(\mathbf{q}, t)
$$

where

$$
\hat{F}_{\alpha \beta \gamma}\left(\mathbf{k}^{\prime}, \mathbf{p}, \mathbf{q}, 2 \Omega t\right)=\sum_{j=1}^{4} \hat{F}_{\alpha \beta \gamma}^{j}\left(\mathbf{k}^{\prime}, \mathbf{p}, \mathbf{q}, 2 \Omega t\right)=\sum_{j=1}^{4} \hat{f}^{j}\left(\mathbf{k}^{\prime}, \mathbf{p}, \mathbf{q}, 2 \Omega t\right) Q_{\alpha \beta \gamma}^{j}\left(\mathbf{k}^{\prime}, \mathbf{p}, \mathbf{q}\right) .
$$

We stress that $k_{3}=0$ implies that we have only four terms in the summation. Since $k_{3}=p_{3}+q_{3}=0$, we obtain

$$
\begin{aligned}
\hat{f}^{1}(\mathbf{k}, \mathbf{p}, \mathbf{q}, 2 \Omega t) & =\cos \left(\frac{p_{3}}{p} 2 \Omega t\right) \cos \left(\frac{q_{3}}{q} 2 \Omega t\right)=\cos \left(\frac{p_{3}}{p} 2 \Omega t\right) \cos \left(\frac{p_{3}}{q} 2 \Omega t\right), \\
\hat{f}^{2}(\mathbf{k}, \mathbf{p}, \mathbf{q}, 2 \Omega t) & =\cos \left(\frac{p_{3}}{p} 2 \Omega t\right) \sin \left(\frac{q_{3}}{q} 2 \Omega t\right)=-\cos \left(\frac{p_{3}}{p} 2 \Omega t\right) \sin \left(\frac{p_{3}}{q} 2 \Omega t\right), \\
\hat{f}^{3}(\mathbf{k}, \mathbf{p}, \mathbf{q}, 2 \Omega t) & =\sin \left(\frac{p_{3}}{p} 2 \Omega t\right) \cos \left(\frac{q_{3}}{q} 2 \Omega t\right)=\sin \left(\frac{p_{3}}{p} 2 \Omega t\right) \cos \left(\frac{p_{3}}{q} 2 \Omega t\right), \\
\hat{f}^{4}(\mathbf{k}, \mathbf{p}, \mathbf{q}, 2 \Omega t) & =\sin \left(\frac{p_{3}}{p} 2 \Omega t\right) \sin \left(\frac{q_{3}}{q} 2 \Omega t\right)=-\sin \left(\frac{p_{3}}{p} 2 \Omega t\right) \sin \left(\frac{p_{3}}{q} 2 \Omega t\right) .
\end{aligned}
$$

In the limit $\mu=\Omega t \gg 1$, Eq. (3.2) has a separation of time scales and can be further simplified. Since $\hat{f}^{2}$ and $\hat{f}^{3}$ are expressed as sums of sin functions only, these terms make no contribution to the dynamics on slow time scale (no resonances). On the other hand, the functions $\hat{f}^{1}$ and $\hat{f}^{4}$ are expressed as sums of cos functions. Then resonances contributing to the slow time scale dynamics are possible only in the case $p=q$. Combining these terms, we obtain

$$
\begin{array}{r}
\hat{f}^{1}\left(\mathbf{k}^{\prime}, \mathbf{p}, \mathbf{q}, 2 \Omega t\right) Q_{\alpha \beta \gamma}^{1}\left(\mathbf{k}^{\prime}, \mathbf{p}, \mathbf{q}\right) u_{\beta}(\mathbf{p}, t) u_{\gamma}(\mathbf{q}, t)+ \\
\hat{f}^{4}\left(\mathbf{k}^{\prime}, \mathbf{p}, \mathbf{q}, 2 \Omega t\right) Q_{\alpha \beta \gamma}^{4}\left(\mathbf{k}^{\prime}, \mathbf{p}, \mathbf{q}\right) u_{\beta}(\mathbf{p}, t) u_{\gamma}(\mathbf{q}, t)= \\
=\cos ^{2}\left(\frac{p_{3}}{p} 2 \Omega t\right) P_{\alpha \beta \gamma}(\mathbf{k}) u_{\beta}(\mathbf{p}, t) u_{\gamma}(\mathbf{q}, t)-\sin ^{2}\left(\frac{p_{3}}{p} 2 \Omega t\right) P_{\alpha \beta \gamma}(\mathbf{k}) u_{\beta}(\mathbf{p}, t) u_{\gamma}(\mathbf{q}, t)= \\
=\cos \left(\frac{p_{3}}{p} 4 \Omega t\right) P_{\alpha \beta \gamma}(\mathbf{k}) u_{\beta}(\mathbf{p}, t) u_{\gamma}(\mathbf{q}, t) .
\end{array}
$$

In the derivation of Eq. (3.5) we have used the fact that $Q_{\alpha \beta \gamma}^{4}(\mathbf{k}, \mathbf{p}, \mathbf{q})=P_{\alpha \beta \gamma}(\mathbf{k})$ if $k_{3}=0$, $p=q$. The above calculation shows that

$$
\begin{gathered}
-\frac{i}{2} \sum_{\mathbf{p}^{\prime}+\mathbf{q}^{\prime}=\mathbf{k}^{\prime}, p_{3}+q_{3}=0} \hat{F}_{\alpha \beta \gamma}\left(\mathbf{k}^{\prime}, \mathbf{p}, \mathbf{q}, 2 \Omega t\right) u_{\beta}(\mathbf{p}, t) u_{\gamma}(\mathbf{q}, t)= \\
-\frac{i}{2} \sum_{\mathbf{p}^{\prime}+\mathbf{q}^{\prime}=\mathbf{k}^{\prime}, p_{3}=q_{3}=0} P_{\alpha \beta \gamma}\left(\mathbf{k}^{\prime}\right) u_{\beta}\left(\mathbf{p}^{\prime}, t\right) u_{\gamma}\left(\mathbf{q}^{\prime}, t\right)+R(\Omega t, \mathbf{u}, \mathbf{u})
\end{gathered}
$$


where $R(\Omega t, \mathbf{u}, \mathbf{u})$ denotes terms evolving on a fast time scale only (without resonances contributing to the dynamics on the slow time scale). Finally, Eq. (3.2) becomes

$$
\frac{\partial}{\partial t} u_{\alpha}\left(\mathbf{k}^{\prime}, t\right)+\nu k^{2} u_{\alpha}\left(\mathbf{k}^{\prime}, t\right)=-\frac{i}{2} \sum_{\mathbf{p}^{\prime}+\mathbf{q}^{\prime}=\mathbf{k}^{\prime}, p_{3}=q_{3}=0} P_{\alpha \beta \gamma}\left(\mathbf{k}^{\prime}\right) u_{\beta}\left(\mathbf{p}^{\prime}, t\right) u_{\gamma}\left(\mathbf{q}^{\prime}, t\right)+R(\Omega t, \mathbf{u}, \mathbf{u})(3.7)
$$

where $R(\Omega t, \mathbf{u}, \mathbf{u})$ is a rapidly oscillating term

$$
\left|\frac{1}{T} \int_{0}^{T} R(\Omega t, \mathbf{u}, \mathbf{u}) d t\right| \leq \frac{c o n s t}{\Omega T} \rightarrow 0
$$

for $\Omega T>>1$. Eq. (3.7) written in physical space has the form

$$
\begin{array}{r}
\frac{\partial}{\partial t} \overline{\mathbf{u}}-\nu \nabla^{2} \overline{\mathbf{u}}+\overline{\mathbf{u}} \cdot \nabla \overline{\mathbf{u}}=-\nabla \bar{p}+R(\Omega t, \mathbf{u}, \mathbf{u}), \\
\nabla \cdot \overline{\mathbf{u}}=0 .
\end{array}
$$

Thus, up to the rapidly oscillating terms $R(\Omega t, \mathbf{u}, \mathbf{u})$, the vertically averaged velocity $\overline{\mathbf{u}}=\overline{\mathbf{U}}$ satisfies the 2D-3C Navier-Stokes equations (3.9) which are averaged in $x_{3}$ initial data. Therefore, we obtain

The analog of the Taylor-Proudman theorem for time-dependent flows:

The vertically averaged component $\overline{\mathbf{U}}$ of the exact solution $\mathbf{U}$ of $3 D-3 C$ Euler/Navier-Stokes equations with initial data $\mathbf{U}_{0}$ is close to the exact solution of 2D-3C Euler/Navier-Stokes equations $\tilde{\mathbf{U}}$ with initial data $\overline{\mathbf{U}}_{0}$ averaged in $x_{3}$. The difference $\mathbf{V}=\mathbf{U}-\tilde{\mathbf{U}}$ between the full $3 D-3 C$ velocity $\mathbf{U}$ and $2 D-3 C$ velocity $\tilde{\mathbf{U}}$ corresponding to the vertically averaged initial data is, however, not small.

A mathematically rigorous formulation and proof of the analog of the Taylor-Proudman theorem for time-dependent flows (including error estimates) are presented in Babin, Mahalov and Nicolaenko ${ }^{27}$.

We now present an alternative development of the time-dependent analog of the TaylorProudman theorem based on physical arguments. Both computations ${ }^{5-9}$ and experiments ${ }^{1-4}$ have noted an increase in integral lengthscales along the rotation axis relative to those in non-rotating turbulence. An increase in the integral lengthscales has been thought to be a prelude to a Taylor-Proudman reorganization to two-dimensional turbulence. It is reasonable to conjecture that at zero Rossby number the only active triads in the asymptotic 
state are those which are invariant under these scalings. This is called the selection principle for triad interactions in the asymptotic state. Clearly, 2D-3C interactions corresponding to $p_{3}=q_{3}=0$ are invariant under scalings of $p_{3}, q_{3}$ in a trivial way. On the other hand, in the asymptotic state of rotating homogeneous turbulence interactions are restricted to pairs of wavevectors satisfying the resonance conditions ${ }^{28,23}$.

$$
\pm \frac{p_{3}}{p} \pm \frac{q_{3}}{q}=\frac{k_{3}}{k}
$$

One can easily find solutions of the resonance equation (3.10) obeying the selection principle. The solutions are $\left\{q_{3}=0, k=p\right\},\left\{p_{3}=0, k=q\right\}$ and $\left\{k_{3}=0, p=q\right\}$. As shown above, the interactions $\left\{k_{3}=0, p=q\right\}$ make no contribution to the dynamics on slow time scale. Two other resonance conditions can be written in equivalent form $\left\{q_{3}=0, k_{1}^{2}+k_{2}^{2}=p_{1}^{2}+p_{2}^{2}\right\}$ and $\left\{p_{3}=0, k_{1}^{2}+k_{2}^{2}=q_{1}^{2}+q_{2}^{2}\right\}$. Clearly, they are invariant under the process of an increase in integral lengthscales along the rotation axis relative to those in the horizontal plane $\left(k_{3} \rightarrow \xi k_{3}, p_{3} \rightarrow \xi p_{3}, q_{3} \rightarrow \xi q_{3}\right)$. Thus the only active triads in the asymptotic state are $\left\{q_{3}=0, k_{1}^{2}+k_{2}^{2}=p_{1}^{2}+p_{2}^{2}\right\}$ and $\left\{p_{3}=0, k_{1}^{2}+k_{2}^{2}=q_{1}^{2}+q_{2}^{2}\right\}$. The condition $p_{3}=0$ simply means that the first entry in the bi-linear form is replaced by its averaged (vertical) value. Similarly, the condition $q_{3}=0$ implies that the second entry is replaced by its averaged value. Therefore, the scale-invariant equations valid in the asymptotic limit $\mu=\Omega t \rightarrow+\infty$ have the form

$$
\frac{\partial \mathbf{u}}{\partial t}=\mathbf{B}(\overline{\mathbf{u}}, \mathbf{u})+\mathbf{B}(\mathbf{u}, \overline{\mathbf{u}}) . \quad \nabla \cdot \mathbf{u}=0
$$

Here $\overline{\mathbf{u}}$ denotes vertical averaging of velocity. Applying the operation of vertical averaging to Eq. (3.11) and using the fact that $\overline{\mathbf{B}(\overline{\mathbf{u}}, \mathbf{u})+\mathbf{B}(\mathbf{u}, \overline{\mathbf{u}})}=\mathbf{B}(\overline{\mathbf{u}}, \overline{\mathbf{u}})+\mathbf{B}(\overline{\mathbf{u}}, \overline{\mathbf{u}})=-\overline{\mathbf{u}} \cdot \nabla \overline{\mathbf{u}}$, we conclude that $\overline{\mathbf{u}}=\overline{\mathbf{U}}$ satisfies the classical 2D-3C Euler equations.

The analog of the Taylor-Proudman theorem for time dependent flows provides a clear picture on the reduction of dimensionality at the limit $\Omega t \rightarrow \infty$. The flow is threedimensional when the $R$ term in Eq. (3.9) is significant (no 'split' of velocity into $\overline{\mathbf{U}}$ and V) while the flow is quasi-two dimensional when $R$ is negligible ('split' of velocity into $\overline{\mathbf{U}}$ and V). The error estimate, Eq. (3.8), can be used to obtain the value of critical $\mu^{*}$ when such a transition takes place. 
We are now in a position to present a physical picture when the time-dependent flow satisfies the analog of the Taylor-Proudman theorem for time dependent flows. There are many two-dimensional layers. If vertically averaged, then the dynamic equations for $\overline{\mathbf{U}}$ are governed by the 2D Navier-Stokes equations with fast-time scale corrections (Eq. 3.7). In this case, the direction of the energy transfer for $\overline{\mathbf{U}}$ is from direct to inverse cascade within each pancake. These layers, however, are not isolated among themselves. For the Euler equations, the $x_{3}$-dependent component $\mathbf{V}$ is not small even at zero Rossby number as shown in Babin, Mahalov and Nicolaen $\mathrm{ko}^{27}$. The evolution equation for $\mathbf{V}$ satisfies a 'passive vector' equation that is driven by $\overline{\mathbf{U}}$. This equation couples these two-dimensional layers and is responsible for the (suppressed) direct energy transfer from large to small scales. The energy spectrum in this case will be discussed in Section 5.

\section{The Poincaré transformed energy transfer equation}

In this section the EDQNM closure is applied to the Poincaré transformed Navier-Stokes equations written in terms of the Poincaré velocity $\mathbf{u}$. Following the standard EDQNM closure procedure, the triple Poincaré velocity correlation is expressed in terms of second order moments. The details are presented in the Appendix. The novelty of our approach is that the EDQNM closure is applied to the Poincaré transformed equations which shows the need for a heuristic approximation for the effects of rotation in the third order moment equations. Lesieur ${ }^{17}$ points out that choice of eddy damping is more difficult in problems where waves interact with turbulence. The previous EDQNM analysis of rotating turbulence requires three evolution equations for second order moments [Cambon and Jacquin ${ }^{10}$ ]. The Coriolis effect, $\Omega$, only appears in one of them. As a result, there are two possibilities on the memory integral. The EDQNM1 version of Cambon and Jacquin ${ }^{10}$ does not include $\Omega$ in the 'damping rate'. On the other hand, $\Omega$ is introduced explicitly into in their EDQNM2 version. They found that these two choices will lead to different results and the latter is needed for the anisotropization mechanisms and to predict a departure from isotropy. Our EDQNM closure is based on the Poincaré transformed Navier-Stokes equations. This Poincaré transformed equation has the same spectral structure as that of the original one 
(with $\Omega$ introduced explicitly into the generalized projection operator) and has a unique memory integral.

The EDQNM closure of the Poincaré transformed equation, presented in detail in the Appendix, provides a framework to study the observed suppression of energy transfer when turbulence is subject to rotation. We assume isotropy of the energy spectrum when examining the energy transfer process. Experiments ${ }^{1-4}$ and $\mathrm{DNS}^{5-9}$ of rotating homogeneous turbulence established that although the rotation increased the anisotropy of the lengthscales, the three principal components of the Reynolds stress tensor remain nearly equal provided that the initial state is isotropic. Thus, our assumption is consistent with these findings.

The isotropy assumption leads to

$$
S_{\alpha \beta}(\mathrm{k}, t)=\frac{1}{2} U(k, t) P_{\alpha \beta}(\mathrm{k}),
$$

where $U(k, t)=\frac{E(k, t)}{4 \pi k^{2}}$ and $E(k, t)$ is the energy spectrum. From the Appendix, the equation for $U(k, t)$ reads

$$
\begin{gathered}
\left(\frac{\partial}{\partial t}+2 \nu k^{2}\right) U(k, t)=\sum_{p}\left(a^{\prime}(k, p, q, \Omega, t) U(p, t) U(q, t)+\right. \\
\left.b^{\prime}(k, p, q, \Omega, t) U(k, t) U(q, t)+c^{\prime}(k, p, q, \Omega, t) U(k, t) U(p, t)\right),
\end{gathered}
$$

where $\mathbf{k}=\mathbf{p}+\mathbf{q}$ is understood. The geometrically determined coefficients $a^{\prime}(k, p, q, \Omega, t)$, $b^{\prime}(k, p, q, \Omega, t)$ and $c^{\prime}(k, p, q, \Omega, t)$ are given in the Appendix. We note that they contain $f$ and the memoral integral $\theta$ (see (A.5)). In order to evaluate these integrals, we expand $f$ in a sum of $\sin$ and $\cos$ using the identities of a kind

$\cos \phi \cos \psi \cos \chi=\frac{1}{4}(\cos (\phi+\psi+\chi)+\cos (\phi+\psi-\chi)+\cos (\phi-\psi+\chi)+\cos (\phi-\psi-\chi))$, Odd terms in $t$ can be expanded in sums of $\sin$ functions. For example, $\sin \phi \cos \psi \cos \chi=\frac{1}{4}(\sin (\phi+\psi+\chi)+\sin (\phi+\psi-\chi)+\sin (\phi-\psi+\chi)+\sin (\phi-\psi-\chi))$.

These identities and the following identities

$$
\begin{gathered}
\int e^{a x} \cos (b x) d x=\frac{e^{a x}}{a^{2}+b^{2}}(a \cos (b x)+b \sin (b x)), \\
\int e^{a x} \sin (b x) d x=\frac{e^{a x}}{a^{2}+b^{2}}(a \sin (b x)-b \cos (b x))
\end{gathered}
$$


are now used to obtain analytical expressions for the coefficients $\theta$ (see (A.5)).

Let $\phi=\frac{p_{3}+q_{3}}{|\mathbf{p}+\mathbf{q}|}, \psi=\frac{p_{3}}{p}$ and $\chi=\frac{q_{3}}{q}$. Then we obtain

$$
\begin{gathered}
\int_{0}^{t} e^{-\left[\nu\left(k^{2}+p^{2}+q^{2}\right)+\mu_{k p q}\right](t-\tau)} \cos (\phi 2 \Omega \tau) \cos (\psi 2 \Omega \tau) \cos (\chi 2 \Omega \tau) d \tau=\frac{1}{4}\left(\theta_{++}(k, p, q, \Omega . \Omega t)+\right. \\
\left.\theta_{+-}(k, p, q, \Omega, \Omega t)+\theta_{-+}(k, p, q, \Omega, \Omega t)+\theta_{--}(k, p, q, \Omega, \Omega t)\right), \\
\int_{0}^{t} e^{-\left[\nu\left(k^{2}+p^{2}+q^{2}\right)+\mu_{k p q}\right](t-\tau)} \cos (\phi 2 \Omega \tau) \sin (\psi 2 \Omega \tau) \sin (\chi 2 \Omega \tau) d \tau=\frac{1}{4}\left(\theta_{+-}(k, p, q, \Omega, \Omega t)+\right. \\
\left.\theta_{-+}(k, p, q, \Omega, \Omega t)-\theta_{++}(k, p, q, \Omega, \Omega t)-\theta_{--}(k, p, q, \Omega, \Omega t)\right), \\
\int_{0}^{t} e^{-\left[\nu\left(k^{2}+p^{2}+q^{2}\right)+\mu_{k p q}\right](t-\tau)} \sin (\phi 2 \Omega \tau) \cos (\psi 2 \Omega \tau) \sin (\chi 2 \Omega \tau) d \tau=\frac{1}{4}\left(\theta_{+-}(k, p, q, \Omega . \Omega t)+\right. \\
\left.\theta_{--}(k, p, q, \Omega, \Omega t)-\theta_{++}(k, p, q, \Omega, \Omega t)-\theta_{-+}(k, p, q, \Omega, \Omega t)\right) \\
\int_{0}^{t} e^{-\left[\nu\left(k^{2}+p^{2}+q^{2}\right)+\mu_{k p q}\right](t-\tau)} \sin (\phi 2 \Omega \tau) \sin (\psi 2 \Omega \tau) \cos (\chi 2 \Omega \tau) d \tau \\
\left.\theta_{-+}(k, p, q, \Omega, \Omega t)-\theta_{++}(k, p, q, \Omega, \Omega t)-\theta_{+-}(k, p, q, \Omega, \Omega t)\right) .
\end{gathered}
$$

The EDQNM relaxation time scales $\theta_{ \pm \pm}(k, p, q, \Omega, \Omega t)$ and $\hat{\theta}_{ \pm \pm}(k, p, q, \Omega, \Omega t)$ are given by

$$
\begin{array}{r}
\theta_{ \pm \pm}(k, p, q, \Omega, \Omega t)=\frac{1}{4} \\
\times\left\{\frac{(\phi \pm \psi \pm \chi) 2 \Omega \sin ((\phi \pm \psi \pm \chi) 2 \Omega t)+\left[\nu\left(k^{2}+p^{2}+q^{2}\right)+\mu_{k p q}\right] \cos ((\phi \pm \psi \pm \chi) 2 \Omega t)}{\left[\nu^{2}\left(k^{2}+p^{2}+q^{2}\right)+\mu_{k p q}\right]^{2}+(\phi \pm \psi \pm \chi)^{2} \Omega^{2}}\right. \\
\left.-\exp \left[-\left[\nu\left(k^{2}+p^{2}+q^{2}\right)+\mu_{k p q}\right] t\right] \frac{\left[\nu\left(k^{2}+p^{2}+q^{2}\right)+\mu_{k p q}\right]}{\left[\nu^{2}\left(k^{2}+p^{2}+q^{2}\right)+\mu_{k p q}\right]^{2}+(\phi \pm \psi \pm \chi)^{2} \Omega^{2}}\right\}
\end{array}
$$

and

$$
\begin{array}{r}
\hat{\theta}_{ \pm \pm}(k, p, q, \Omega, \Omega t)=\frac{1}{4} \\
\times\left\{\frac{(\phi \pm \psi \pm \chi) 2 \Omega \cos ((\phi \pm \psi \pm \chi) 2 \Omega t)+\left[\nu\left(k^{2}+p^{2}+q^{2}\right)+\mu_{k p q}\right] \sin ((\phi \pm \psi \pm \chi) 2 \Omega t)}{\left[\nu^{2}\left(k^{2}+p^{2}+q^{2}\right)+\mu_{k p q}\right]^{2}+(\phi \pm \psi \pm \chi)^{2} \Omega^{2}}\right. \\
\left.-\exp \left[-\left[\nu\left(k^{2}+p^{2}+q^{2}\right)+\mu_{k p q}\right] t\right] \frac{(\phi \pm \psi \pm \chi) 2 \Omega}{\left[\nu^{2}\left(k^{2}+p^{2}+q^{2}\right)+\mu_{k p q}\right]^{2}+(\phi \pm \psi \pm \chi)^{2} \Omega^{2}}\right\}
\end{array}
$$


Since the eddy damping is much greater than the molecular viscosity, the EDQNM time scale for the triple velocity decorrelation can be simplified to

$$
\begin{array}{r}
\theta_{ \pm \pm}(k, p, q, \Omega, \Omega t) \approx \frac{1}{4}\left\{\frac{(\phi \pm \psi \pm \chi) 2 \Omega \sin ((\phi \pm \psi \pm \chi) 2 \Omega t)+\mu_{k p q} \cos ((\phi \pm \psi \pm \chi) 2 \Omega t)}{\mu_{k p q}^{2}+(\phi \pm \psi \pm \chi)^{2} \Omega^{2}}\right. \\
\left.-\exp \left[-\mu_{k p q} t\right] \frac{\mu_{k p q}}{\mu_{k p q}^{2}+(\phi \pm \psi \pm \chi)^{2} \Omega^{2}}\right\}(4.6)
\end{array}
$$

and

$$
\begin{array}{r}
\hat{\theta}_{ \pm \pm}(k, p, q, \Omega, \Omega t) \approx \frac{1}{4}\left\{\frac{(\phi \pm \psi \pm \chi) 2 \Omega \cos ((\phi \pm \psi \pm \chi) 2 \Omega t)+\mu_{k p q} \sin ((\phi \pm \psi \pm \chi) 2 \Omega t)}{\mu_{k p q}^{2}+(\phi \pm \psi \pm \chi)^{2} \Omega^{2}}\right. \\
\left.-\exp \left[-\mu_{k p q} t\right] \frac{(\phi \pm \psi \pm \chi) 2 \Omega}{\mu_{k p q}^{2}+(\phi \pm \psi \pm \chi)^{2} \Omega^{2}}\right\}(4.7)
\end{array}
$$

They characterize the relaxation of $\langle u(k) u(p) u(q)>$ toward a quasi-equilibrium by nonlinear transfer and molecular viscosity. Rather than solving the EDQNM evolution equations, we will only use information about the triple time scale of velocity decorrelation obtained from this theory as an input for a phenomenological treatment. In the next section we will use this information to deduce the energy spectrum and the spectral eddy viscosity in various asymptotic limits.

\section{$5 \quad$ Phenomenological analysis at asymptotic limits}

In order to infer the form of the inertial-range spectrum, it is necessary to estimate the magnitude for the triple correlations. In general, $\tau_{3}$, the time scale for decay of triple correlations which is responsible for inducing turbulent spectral transfer, may depend on any relevant turbulence parameters. Because energy is conserved by the nonlinear interaction and a local cascade has been assumed, $\epsilon$ is independent of $k$. Local cascade ${ }^{29-31}$ also implies that $\epsilon$ is explicitly proportional to $\tau_{3}$ and depends on the wavenumber and on the power of the omni-directional energy spectrum ${ }^{12}$. A simple dimensional analysis leads to

$$
\epsilon=A^{2} \tau_{3}(k) k^{4} E^{2}(k)
$$

where $A$ is a constant ${ }^{32}$. If the energy-containing range excitation were absent, it would be expected that the nonlinear interaction would build up substantial triple correlations in 
the local dynamical time $\tau_{n l}(k)$. In steady state isotropic turbulence, the only time scale available for $\tau_{3}(k)$ is the nonlinear time scale, $\tau_{n l}(k)$; therefore the Kolmogorov ${ }^{18}$ spectrum is recovered. The equation which determines the spectral eddy viscosity, $\nu_{T}(k)$, is given $\mathrm{by}^{33}$

$$
\epsilon=\nu_{T}(k) S^{2}(k)=\nu_{T}(k) E(k) k^{3}
$$

In our spectral representation, it is convenient to use the spectral Rossby number $R o^{s}(k)=\mu_{k} / \Omega$, which is the ratio of turbulence (nonlinear eddy turn-over) time scale and rotation time scale. For a given inertial Rossby number, we can define the cases of strong rotation when $R o^{s} \ll 1$ and the weak rotation when $R o^{s} \gg 1$.

We now consider the number of the non-dimensional parameters needed. For the turbulence in equilibrium the Rossby number is the only relevant parameter controlling the effects of rotation on the flow. However, for the non-equilibrium situation, new non-dimensional parameters $\mu=\Omega t$ and $\sigma=\mu_{k} t$ are required. They correspond to non-equilibrium effects at long and short time scales, respectively.

We have shown that the parameter $\mu=\Omega t$ is introduced into the time scale of the triple velocity product as the arguments of sin and cos functions. These terms are clearly responsible for the scrambling of the energy transfer and do not go to zero as $t \rightarrow \infty$. As a result, the values of the time scale of the triple velocity product will oscillate; and this oscillation can be removed only by a long time average. For a turbulent flow with a given initial Rossby number (therefore, the rotation rate $1 / \Omega$ ), the non-dimensional parameter $\mu$ will evolve in three phases: (1) $\mu \rightarrow 0$; (2) $\mu$ bounded; (3) $\mu \rightarrow \infty$. In addition, $\sigma=\mu_{k} t$ is introduced in the second term in both $\theta_{ \pm}$and $\hat{\theta}_{ \pm}$as a damping factor. This damping term is significant initially but it will go to zero as $t \rightarrow \infty$.

It is clear that the general treatment of this problem is rather complicated. When a resonance condition, say $\phi+\psi+\chi=0$, is satisfied, the corresponding decorrelation time $\theta_{++}$is simply the nonlinear time scale $\tau_{n l}=1 /\left[k^{3} E(k)\right]^{1 / 2}$ with a damping factor $\left[1-\exp \left[-\left[\mu_{k p q}\right] t\right]\right]$ for short time scale.

The non-resonance condition needs to be examined with care. Asymptotic limits of strong and weak rotation offer considerable simplification. These limits correspond to small 
(spectral or initial) and large Rossby numbers, respectively.

For the small Rossby number case, we have

$$
\theta_{ \pm \pm}(k, p, q, \Omega, \Omega t) \approx \frac{\sin ((\phi \pm \psi \pm \chi) 2 \Omega t)}{(\phi \pm \psi \pm \chi) \Omega}-\exp \left[-\mu_{k p q} t\right] \frac{\mu_{k p q}}{(\phi \pm \psi \pm \chi)^{2} \Omega^{2}}
$$

and

$$
\hat{\theta}_{ \pm \pm}(k, p, q, \Omega, \Omega t) \approx \frac{\cos ((\phi \pm \psi \pm \chi) 2 \Omega t)}{(\phi \pm \psi \pm \chi) \Omega}-\exp \left[-\mu_{k p q} t\right] \frac{1}{(\phi \pm \psi \pm \chi) \Omega}
$$

Alternatively, for large Rossby number, one can show that

$$
\theta_{ \pm \pm}(k, p, q, \Omega, \Omega t) \approx \frac{\cos ((\phi \pm \psi \pm \chi) 2 \Omega t)}{\mu_{k p q}}-\exp \left[-\mu_{k p q} t\right] \frac{1}{\mu_{k p q}}
$$

and

$$
\hat{\theta}_{ \pm \pm}(k, p, q, \Omega, \Omega t) \approx \frac{\sin ((\phi \pm \psi \pm \chi) 2 \Omega t)}{\mu_{k p q}}-\exp \left[-\mu_{k p q} t\right] \frac{(\phi \pm \psi \pm \chi) 2 \Omega}{\mu_{k p q}^{2}} .
$$

\subsection{Disruption of homogeneous isotropic turbulence by rotation}

We now focus on the short time behavior of the decorrelation time scale for the triple velocity products.

There are two different situations. First, the time under consideration is so short $(t \rightarrow 0)$ that both $\theta_{ \pm \pm} \rightarrow 0$ and $\hat{\theta}_{ \pm \pm} \rightarrow 0$ by definition in Eqs. (4.6)-(4.7). When the rotation is also very strong, it is called the Rapid Distortion Theory (RDT) limit ${ }^{34-36}$. To make contact with RDT, we rewrite $\mu=t^{*} / t_{\Omega}^{*}$, where $t^{*}=\epsilon t / K_{0}$ and $t_{\Omega}^{*}=\epsilon /\left(K_{0} \Omega\right)$. This procedure nondimensionalizes the time scales using the initial turbulent kinetic energy $\left(K_{0}\right)$ and dissipation rate. Note that the inertial Rossby number of the flow is defined as $R o=$ $0.5 \epsilon /\left(K_{0} \Omega\right)$. RDT theory assumes that the nonlinear effects do not have time to build up under the conditions of very short time and strong system rotation. Specifically, the RDT limit is valid when the initial Rossby number $R o \ll 1$ and the time normalized by initial turbulent time $t^{*} \ll 1$. 
We now consider the second case where the nonlinear effects become important but time $t^{*}$ is still short. Again, two distinct physical situations are included in this limit: (a) weak rotation; (b) strong rotation (we call it 'disruption' as an extension of the RDT limit). In the 'disruption' limit the rotation is very strong. In a fixed time interval, $\Omega$ is very large. However, the time $t$ is very short so that $\Omega t$ is bounded.

For the weak rotation case (large $R o^{s}$ ), we have

$$
\theta \approx \hat{\theta} \sim\left[1-e^{-\mu_{k p q} t}\right] \times \frac{1}{\mu_{k p q}} .
$$

Similarly, for the strong rotation case, one has

$$
\theta \sim\left[1-e^{-\mu_{k p q} t}\right] \times \frac{\sin (2 \Omega t)}{\Omega},
$$

and

$$
\hat{\theta} \sim\left[1-e^{-\mu_{k p q} t}\right] \times \frac{\cos (2 \Omega t)}{\Omega} .
$$

These relations form a basis for constructing implicit equations for the energy spectra and spectral eddy viscosity in non-equilibrium turbulent flows. Such flows are deferred to a future study.

\subsection{Long-time dynamics of uniformly rotating fluids}

We now consider the limit of long-time dynamics. Here, the long time limit means that the time $t$ is large enough so that the damping terms in $\theta$ and $\hat{\theta}$ can be neglected. Again, we must consider two physically distinct situations: strong and weak rotation. In the weak rotation case we find that the decay rate of triple velocity correlations is given by the turbulence nonlinear time scale $\tau_{n l}$. Therefore, at leading order, the energy spectrum and spectral viscosity are the same as that of the non-rotating case. The rotation effects can be introduced trivially here by keeping the next order correction for rotation in $\theta$ and $\hat{\theta}$. We omit this analysis here.

In the the strong rotation case, the dependence of $\theta_{ \pm \pm}$and $\hat{\theta}_{ \pm \pm}$on $\Omega$ should be considered with care. The time scale for $\tau_{3}(k)$, the decorrelation of the triple velocity product, is 
again the controlling parameter influencing the energy transfer process. When a resonance condition, say $\phi+\psi+\chi=0$, is satisfied, the corresponding decorrelation time $\theta_{++}$is simply the nonlinear time scale $\tau_{n l}=1 /\left[k^{3} E(k)\right]^{1 / 2}$. In the mean time, the other contributions to this time scale at this given condition, $\theta_{+-}, \theta_{-+}, \theta_{--}$are of order

$$
\theta \sim \theta_{+-} \sim \theta_{-+} \sim \theta_{--} \sim \frac{\sin (2 \Omega t)}{\Omega} .
$$

Similarly, we find that

$$
\hat{\theta}_{\Omega} \sim \frac{\cos (2 \Omega t)}{\Omega}
$$

In order to remove the phase from Eqs (5.10)-(5.11), we introduce the long time averaged time scale which determines the decorrelation of triple velocity products at a small spectral Rossby number $(\Omega t \rightarrow \infty)$ :

$$
\tau_{3}=\bar{\theta}_{\Omega}=\sqrt{\theta_{\Omega}^{2}+\hat{\theta}_{\Omega}^{2}} \sim 1 / \Omega
$$

Therefore, in the regime of high Reynolds numbers and low Rossby numbers, rotating turbulence is characterized by two disparate time-scales: a short time scale associated with the rotation frequency $\tau_{\Omega}=1 / \Omega$ and a nonlinear time scale. Our EDQNM time scale discussed above provides an additional support for the choice of $\tau_{\Omega}=1 / \Omega$ in the phenomenological analysis of Zhou ${ }^{11}$. This is the key assumption in his application of the extended phenomenology of Kraichnan ${ }^{12}$ and Mattheaus and Zhou ${ }^{32}$ to isotropic turbulence subject to rotation. This is also the basic assumption in the large-eddy simulation (LES) of strong rotating turbulence of Squire et al. ${ }^{9}$ where they argued that the time scale of the triple velocity correlation is proportional to $\tau_{\Omega}=1 / \Omega$. Squires et al. ${ }^{9}$ assumed that the correlation time of the nonlinear triadic interactions is directly proportional to the short time scale $\tau_{\Omega}$. The predictions of the asymptotic decay for the kinetic energy in rotating turbulence, based on this crucial assumption on the time scale, have been confirmed within a few percent by their LES runs for times of order $O\left(10^{3}\right)$ of initial turbulence. We find that a direct application of $\tau_{3}=\tau_{\Omega}$ resulted the energy spectrum for turbulence subject to strong rotation:

$$
E(k)=C_{\Omega}(\Omega \epsilon)^{1 / 2} k^{-2}
$$


In general, Zhou ${ }^{11}$ has pointed out that there is a need to incorporate both rotating and eddy-turn-over time scales. We make use of the viewpoint that the lifetime of triple correlations in rotating turbulence might be more accurately treated by taking into account the possibility that these correlations decay because of the influence of both wave propagation and nonlinear triadic interactions ${ }^{32}$. The simple choice

$$
\frac{1}{\tau_{3}(k)}=\frac{1}{\tau_{n l}(k)}+\frac{1}{\tau_{\Omega}(k)}
$$

satisfies the appropriate limiting cases: $\tau_{3}(k) \rightarrow \tau_{n l}$ without rotation and $\tau_{3}(k) \rightarrow \tau_{\Omega}$ with strong rotation. The generalized inertial range energy spectrum is

$$
E(k)=Z^{2} A^{-4 / 3} \epsilon^{2 / 3} k^{-5 / 3},
$$

where $Z$ is given by ${ }^{11,32}$

$$
Z=\frac{1}{2}\left(\sqrt{Y}+\sqrt{-Y+2 \sqrt{Y^{2}+4 Z_{0}}}\right)
$$

where

$$
Y=\sqrt[3]{\frac{1}{2}+\sqrt{\frac{1}{4}+\left(\frac{4 Z_{0}}{3}\right)^{3}}}+\sqrt[3]{\frac{1}{2}-\sqrt{\frac{1}{4}+\left(\frac{4 Z_{0}}{3}\right)^{3}}}
$$

The parameters $A=C_{K}^{-3 / 4}$ (Matthaeus and Zhou ${ }^{32}$ ), $k_{\Omega}=\left(\Omega^{3} / \epsilon\right)^{1 / 2}$ and $Z_{0}=$ $A^{2 / 3} \frac{\Omega}{\left(\epsilon k^{2}\right)^{1 / 3}}=\left[\frac{A k_{\Omega}}{k}\right]^{2 / 3}$. The strong rotation limit then leads to $C_{\Omega}=1 / A=1.22-1.87$ for the typical range of Kolmogorov constant. These equations reduce to the non-rotating Kolmogorov "-5/3" spectrum when $Z_{0} \rightarrow 0$ (so that $Z \rightarrow 1$ ), and to our rotating modified “-2" spectrum when $Z_{0} \rightarrow \infty$ (so that $\left.Z \rightarrow Z_{0}^{1 / 4}\right)$. Fig. 1 illustrates spectra obtained this way with $\epsilon=1$ and $A=0.7$ (we have chosen $C_{K}=1.5$ ), varying the reference rotation rate over values $\Omega=0,10$, and 100 . The zero rotation case is a pure Kolmogorov spectrum while the $\Omega=100$ case is very nearly $k^{-2}$ spectrum. For intermediate rotation rates the spectrum varies smoothly between these two limiting forms, according to the increase of the controlling parameter $Z_{0}$ with increasing ratio $k_{\Omega} / k$.

The rotation dependent eddy viscosity can be estimated ${ }^{11}$ as

$$
\nu_{T}(k)=\nu_{0}\left[\frac{E(k)}{k}\right]^{1 / 2} \frac{1}{1+y^{-1 / 2}},
$$


where $\nu_{0}$ is a constant and $y=k^{3} E(k) / \Omega^{2}$ is defined following Zeman ${ }^{33}$. The inertial range wavenumber $k$ can be related ${ }^{37-38}$ to the turbulent kinetic energy $K$ and dissipation rate $\epsilon$

$$
k=\frac{E(k)}{K^{3 / 2}}\left(3 C_{k} / 2\right)^{3 / 2} .
$$

For the inertial range spectrum, Eq. (5.18) can be rewritten in physical space:

$$
\nu_{T}(x)=\nu_{0}^{\prime} \frac{K^{2}}{\epsilon} \frac{1}{1+0.36 K \Omega / \epsilon},
$$

The eddy viscosity above is only appropriate for homogeneous turbulence without mean velocity gradients. For turbulent flows with mean velocity gradients in a rotating frame, the effect of rotation appears in conjunction with the mean vorticity ${ }^{39}$ and Eq. (5.19) can be extended in a straightforward way by replacing $\Omega$ by $\left(\bar{W}_{i j} \bar{W}_{i j}\right)^{1 / 2}$ (Gatski and Speziale ${ }^{40}$ ). Here $\bar{W}_{i j}=\bar{\omega}_{i j}+\alpha \epsilon_{m j i} \Omega_{m}$ is the extended absolute vorticity tensor defined by Gatski and Speziale ${ }^{40}$ ( $\alpha$ is a constant). The constant $\nu_{0}^{\prime}$ can then be evaluated in homogeneous shear flow where $W_{i j}$ reduces to the uniform shear rate. Such flows are deferred to a future study. We note that the scaling of the eddy viscosity with rotation rate is inconsistent with RDT.

The spectral time scale is an important measurement ${ }^{11}$. The Kolmogorov hypothesis implies that the energy-containing range excitation does not affect energy transfer within the inertial range. Therefore, the average rate of energy dissipation is identified with the rate of spectral energy transfer and the rate of energy input. The nonlinear (or eddy turnover) time-scale, $\tau_{n l}(k) \equiv\left[k^{3} E(k)\right]^{-1 / 2}$, is then equivalent to the spectral transfer time, $\tau_{s}$. Zhou ${ }^{11}$ has shown that

$$
\tau_{s}(k)=\frac{1}{A^{2}} \frac{\left[\tau_{n l}(k)\right]^{2}}{\tau_{\Omega}(k)},
$$

or more generally,

$$
\tau_{s}(k)=\frac{1}{A^{2}} \frac{\tau_{n l}^{2}(k)}{\tau_{3}(k)}
$$

Therefore, the time for the spectral energy transfer is increased to a value greater than $\tau_{n l}$; thus nonlinear energy transfer is suppressed by rotation.

We stress that the long-time averaging procedure here is the same as that introduced for the time-dependent analog of the Taylor-Proudman theorem. This averaging procedure, 
as defined in (3.8), removes the oscillations and 'splits' the velocity fields into two parts. Our analysis suggests that the energy transfer process in the limit of $\Omega t \rightarrow \infty$ and small spectral Rossby number (strong rotation) is as follows. There are inverse energy transfer by $\overline{\mathbf{U}}=\overline{\mathbf{u}}$ with $-5 / 3$ omni-directional energy spectrum. This is the same as the two dimensional turbulence. In the meantime, there is also a direct energy cascade governed by the equation for the passive vector $\mathbf{V}$ introduced in Sec. 3 at $\Omega t \rightarrow \infty$ and $\Omega>>\mu_{k}$. The energy spectrum of the full velocity $\mathbf{U}$ is given by Eqs. (5.15)-(5.17).

\section{Concluding Remarks}

In this paper we presented a general framework to study turbulence subjected to a uniform background rotation based on Green's method. In the analysis presented above we assumed spatial homogeneity of turbulent flows. Although the application of this method in complex geometries alters the integral convolution kernels in the Poincare transformed Navier-Stokes equations, the mathematical procedure is still valid provided that explicit asymptotic expressions for the Green's tensor can be obtained. For example, in the case of a turbulent flow subjected to an uniform background rotation occupying a region $D$, the Green's tensor corresponding to the gyroscopic operator is found from the linear problem

$$
\begin{array}{r}
\frac{\partial}{\partial t} \mathbf{U}-\nu \nabla^{2} \mathbf{U}+2 \Omega \mathbf{J} \mathbf{U}=-\nabla p, \\
\nabla \cdot \mathbf{U}=0 .
\end{array}
$$

Then the Poincaré transformation which relates the fluid velocity $\mathbf{U}$ with the Poincaré velocity variable $\mathbf{u}$ is given by

$$
\mathbf{U}(t, x)=G \mathbf{u}=\int_{D} G(\Omega t, \nu t, x, y) \mathbf{u}(y) d y .
$$

As a result, the Navier-Stokes equations written in terms of the Poincaré velocity variables take the form

$$
\frac{\partial}{\partial t} \mathbf{u}=\mathbf{B}(\Omega t, \nu t, \mathbf{u}, \mathbf{u})
$$

where

$$
\mathbf{B}(\Omega t, \nu t, \mathbf{u}, \mathbf{u})=G^{-1} P\{G \mathbf{u} \times \operatorname{curl} G \mathbf{u}\}
$$


When explicit expression for the gyroscopic operator $G$ can be obtained, the relation between the nonlinear (turbulent), rotation, and viscous time scales in Eq. (6.4) are transparent. In general, explicit expressions are not available. However, for certain domains of interest (e.g. turbulent flow between rotating parallel plates) one can obtain explicit expressions for the gyroscopic operator valid in the asymptotic limits $\mu=\Omega t \rightarrow 0, \mu=\Omega t \rightarrow+\infty$. Then one can apply the analysis presented in this paper.

\section{References}

[1] S.C. Traugott [1958], Influence of solid-body rotation on screen-produced turbulence, NACA Tech. Note 4135.

[2] R.A. Wigeland and H.M. Nagib [1978], Grid-generated turbulence with and without rotation about the streamwise direction, IIT Fluids and Heat Transfer Rep., R78-1, Mlinois Inst. of Tech.

[3] L. Jacquin, O. Lechter, C. Cambon and J. Mathieu [1990], Homogeneous turbulence in the presence of rotation, J. Fluid Mech., 220, 1.

[4] S.V. Veeravalli [1990], An experimental study of the effects of rapid rotation turbulence, Center for Turbulence Research Briefs, Stanford University-NASA Ames Research Center.

[5] J. Bardina, J.H. Ferziger and R.S. Rogallo [1985], Effect of rotation on isotropic turbulence: computation and modelling, J. of Fluid Mech., 154, 321.

[6] C.G. Speziale, N.N. Mansour and R.S. Rogallo [1987], Decay of turbulence in a rapidly rotating frame, Proc. 1987 Center for Turbulence Research Summer Program, Stanford University-NASA Ames Research Center.

[7] N.N. Mansour, C. Cambon and C.G. Speziale [1992], Theoretical and computational study of rotating isotropic turbulence, Studies in Turbulence, edited by T.B. Gatski, S. Sarkar and C.G. Speziale, Springer-Verlag. 
[8] M. Hossain [1994], Reduction in the dimensionality of turbulence due to a strong rotation, Phys. Fluids, 6, 1077.

[9] K.D. Squires, J.R. Chasnov, N.N. Mansour and C. Cambon [1993], Investigation of the asymptotic state of rotating turbulence using LES, Center for Turbulence Research Briefs, 1993, Stanford University-NASA Ames Research Center.

[10] C. Cambon and L. Jacquin [1989], Spectral approach to non-isotropic turbulence subject to rotation, J. Fluid Mech., 202, 295.

[11] Y. Zhou [1995], A phenomenological treatment of rotating turbulence, Phys. Fluids, 7, 2092.

[12] R.H. Kraichnan [1965], Inertial range spectrum of hydromagnetic turbulence, Phys. Fluids 8, 1385 .

[13] H. Poincaré [1910], Sur la précession des corps déformables, Bull. Astronomique, 27, 321.

[14] A. Bershadskii, E. Kit and A. Tsinober [1993], Spontaneous breaking of reflectional symmetry in real quasi-two-dimensional turbulence: stochastic travelling waves and helical solitons in atmosphere and laboratory, Proc. R. Soc. Lond. A, 441, 147.

[15] E.J. Hopfinger, F.K. Browand and Y. Gagne [1982], Turbulence and waves in a rotating tank, J. Fluid Mech., 125, 505.

[16] S.A. Orszag [1970], Analytical theories of turbulence, J. Fluid Mech., 41, 363.

[17] M. Lesieur [1987], Turbulence in Fluids, Martinus Nijhoff Publishers.

[18] A.N. Kolmogorov [1941], The local structure of turbulence in incompressible viscous fluid for very large Reynolds numbers, Dokl. Akad. Nauk SSSR, 30, 299.

[19] O. Ladyzhenskaya [1971], Mathematical theory of viscous incompressible fluids, Gordon and Breach. 
[20] S.L. Sobolev [1954], Ob odnoi novoi zadache matematicheskoi fiziki, Izvestiia Akademii Nauk SSSR, Ser. Matematicheskaia, 18, No. 1, 3.

[21] H.K. Moffatt [1970], Dynamo action associated with random inertial waves in a rotating conducting fluid, J. Fluid Mech., 44, 705.

[22] H.E. Moses [1971], Eigenfunctions of the curl operator, rotationally invariant Helmholtz theorem and applications to electromagnetic theory and fluid mechanics, SIAM J. Appl., 21, 114.

[23] F. Waleffe [1993], Inertial transfers in the helical decomposition, Phys. of Fluids A, 5, 677.

[24] J.-Z. Wu, D. Wu, Y. Zhou and X. Wu [1995], Helical wave decomposition for weakly compressible flows, in preparation.

[25] D.J. Tritton [1977], Physical Fluid Dynamics, Van Nostrand, New York.

[26] C.G. Speziale [1989], Turbulence modeling in noninertial frames of reference, Theor. and Comp. Fluid Dyn., 3.

[27] A. Babin, A. Mahalov and B. Nicolaenko [1995], Global splitting, integrability and regularity of 3D Euler and Navier-Stokes equations for uniformly rotating fluids, in press.

[28] H. Greenspan [1968], The Theory of Rotating Fluids, Cambridge University Press.

[29] Y. Zhou [1993], Degrees of locality of energy transfer in the inertial range, Phys. Fluids A, $5,1092$.

[30] Y. Zhou [1993], Interacting scales and energy transfer in isotropic turbulence, Phys. Fluids A, 5, 2511.

[31] R.H. Kraichnan [1971], Inertial range transfer in two- and three- dimensional turbulence, J. Fluid Mech., 47, 525. 
[32] W.H. Matthaeus and Y. Zhou [1989], Extended inertial range phenomenology of magnetohydrodynamic turbulence, Phys. Fluids B1, 1929.

[33] O. Zeman [1994], A note on the spectra and decay of rotating homogeneous turbulence, Phys. Fluids, 6, 3221.

[34] A.A. Townsend [1976], The structure of turbulent shear flows, Cambridge University Press.

[35] J.C.R. Hunt [1978], A review of the theory of rapidly distorted turbulent flows and its applications, Fluid Dyn. Trans., 9, 121.

[36] W.C. Reynolds [1987], Fundamentals of turbulence for turbulence modeling and simulations, Lecture Notes for Von Karman Institute.

[37] R. Rubinstein, and J.M. Barton [1990], Nonlinear Reynolds stress model and the renormalization group. Phys. Fluids A, 2, 1472.

[38] Y. Zhou, G. Vahala, and S. Thangam [1994], Development of a recursive RNG based turbulence model. Phys. Rev. E, 49, 5195.

[39] C.G. Speziale [1991], Analytical methods for the development of Reynolds stress closures in turbulence, Ann. Rev. Fluid Mech., 23, 107.

[40] T.B. Gatski and C.G. Speziale [1993], On explicit algebraic stress models for complex turbulent flows, J. Fluid Mech., 254, 59. 


\section{A Appendix: EDQNM closure for the Poincaré transformed equations}

We consider the most general term in (2.17). It has the form

$$
\frac{\partial u_{\alpha}}{\partial t}(\mathbf{k}, t)+\nu k^{2} u_{\alpha}(\mathbf{k}, t)=-\frac{i}{2} \sum_{p} f(p, k, \Omega t) Q_{\alpha \beta \gamma}(k, p) u_{\beta}(\mathbf{p}, t) u_{\gamma}(\mathbf{k}-\mathbf{p}, t) .
$$

We define

$$
\begin{array}{r}
S_{\alpha \beta}(\mathbf{k}, t)=<u_{\alpha}(\mathbf{k}, t) u_{\beta}(-\mathbf{k}, t)>, \\
T_{\alpha \beta \gamma}(\mathbf{k}, \mathbf{p}, t)=<u_{\alpha}(\mathbf{k}, t) u_{\beta}(\mathbf{p}, t) u_{\gamma}(-\mathbf{k}-\mathbf{p}, t)>, \\
U_{\alpha \beta \gamma \delta}(\mathbf{k}, \mathbf{p}, \mathbf{q}, t)=<u_{\alpha}(\mathbf{k}, t) u_{\beta}(\mathbf{p}, t) u_{\gamma}(\mathbf{q}, t) u_{\delta}(-\mathbf{k}-\mathbf{p}-\mathbf{q}, t)>.
\end{array}
$$

The tensors $S, T, U$ are called cumulants of the second, third and fourth order, respectively.

Following Orszag ${ }^{16}$, we obtain equations for $S_{\alpha \beta}(\mathbf{k}, t)$ and $T_{\alpha \beta \gamma}(\mathbf{k}, \mathbf{p}, t)$. We have

$$
\begin{array}{r}
\left(\frac{\partial}{\partial t}+2 \nu k^{2}\right) S_{\alpha \beta}(\mathbf{k}, t)= \\
-\frac{i}{2} \sum_{p}\left(f(p, k, \Omega t) Q_{\alpha \rho \sigma}(\mathbf{p}, \mathbf{k}) T_{\beta \rho \sigma}(-\mathbf{k}, \mathbf{p}, t)+f(p,-k, \Omega t) Q_{\beta \rho \sigma}(\mathbf{p},-\mathbf{k}) T_{\alpha \rho \sigma}(\mathbf{k}, \mathbf{p}, t)\right)
\end{array}
$$

and

$$
\begin{aligned}
\left(\frac{\partial}{\partial t}+\nu\left(k^{2}+p^{2}+q^{2}\right)\right) T_{\alpha \beta \gamma}(\mathbf{k}, \mathbf{p}, t)= & -\frac{i}{2} \sum_{r}\left(Q_{\alpha \rho \sigma}(\mathbf{r}, \mathbf{k}) f(r, k, \Omega t) U_{\beta \gamma \rho \sigma}(\mathbf{p}, \mathbf{q}, \mathbf{r}, t)+\right. \\
& Q_{\beta \rho \sigma}(\mathbf{r}, \mathbf{p}) f(r, p, \Omega t) U_{\alpha \gamma \rho \sigma}(\mathbf{k}, \mathbf{q}, \mathbf{r}, t)+ \\
& \left.Q_{\gamma \rho \sigma}(\mathbf{r}, \mathbf{q}) f(r, q, \Omega t) U_{\alpha \beta \rho \sigma}(\mathbf{k}, \mathbf{p}, \mathbf{r}, t)\right) .
\end{aligned}
$$

We then use a quasi-normal assumption and express $U_{\alpha \beta \rho \sigma}(\mathbf{k}, \mathbf{p}, \mathbf{r}, t)$ in terms of $S$ It implies

$$
\begin{array}{r}
\left(\frac{\partial}{\partial t}+\nu\left(k^{2}+p^{2}+q^{2}\right)\right) T_{\alpha \beta \gamma}(\mathbf{k}, \mathbf{p}, t)= \\
-\frac{i}{2}\left(Q_{\alpha \rho \sigma}(-\mathbf{p}, \mathbf{k}) f(-p, k, \Omega t) S_{\beta \rho}(\mathbf{p}, t) S_{\gamma \sigma}(\mathbf{q}, t)+Q_{\alpha \rho \sigma}(-\mathbf{q}, \mathbf{k}) f(-q, k, \Omega t) S_{\beta \sigma}(\mathbf{p}, t) S_{\gamma \rho}(\mathbf{q}, t)+\right. \\
Q_{\beta \rho \sigma}(-\mathbf{k}, \mathbf{p}) f(-k, p, \Omega t) S_{\alpha \rho}(\mathbf{k}, t) S_{\gamma \sigma}(\mathbf{q}, t)+Q_{\beta \rho \sigma}(-\mathbf{q}, \mathbf{p}) f(-\mathbf{q}, \mathbf{p}, \Omega t) S_{\alpha \sigma}(\mathbf{k}, t) S_{\gamma \rho}(\mathbf{q}, t)+ \\
\left.Q_{\gamma \rho \sigma}(-\mathbf{k}, \mathbf{q}) f(-k, q, \Omega t) S_{\alpha \rho}(\mathbf{k}, t) S_{\beta \sigma}(\mathbf{p}, t)+Q_{\gamma \rho \sigma}(-\mathbf{p}, \mathbf{q}) f(-p, q, \Omega t) S_{\alpha \sigma}(\mathbf{k}, t) S_{\beta \rho}(\mathbf{p}, t)\right) .
\end{array}
$$

Before we proceed further, the Eddy Damping approximation must be introduced. For the non-rotating turbulence, it was found that the quasi-normal approximation alone is not satisfactory. In particular, the energy spectrum obtained in evolution would become negative 
valued. Orszag ${ }^{16}$ found that this unphysical behavior may be understood on the basis of improper relaxation time. Formal solution of $T$ from its evolution equation introduces a memory integral. The memory integral should not involve only a viscous cut-off when the Reynolds number is large. Instead, the memory should cut-off because phase correlation between Fourier modes (or eddies) do not persist in the random convention fields of other eddies. The Eddy-Damped Quasi-normal approximation is proposed [Orszag ${ }^{16}$. The essential of the 'Eddy-Damping' is to approximate the fourth-order cumulants neglected by a linear damping term and to enhance the viscous term by $\nu\left(k^{2}+p^{2}+q^{2}\right)+\mu_{k p q}$. The parameter $\mu_{k p q}$, which has the dimension of the inverse time, is the characteristic "decorrelation rate" of the third order moments. Lesieur ${ }^{17}$ stressed that its choice is essential if one want to use the theory for quantitative predictions. For isotropic turbulence, Orszag ${ }^{16}$ recommended that

$$
\mu_{k p q}=\mu_{k}+\mu_{p}+\mu_{q}
$$

where

$$
\mu_{k} \sim\left[k^{3} E(k)\right]^{1 / 2}
$$

is the inverse of the local eddy turn over time. This approximation is known as the EddyDamped Quasi-Normal (EDQN) approximation.

As discussed in detail by Orszag $^{16}$ and Lesieur ${ }^{17}$, the EDQN approximation does not guarantee the realizability (the positiveness of the energy spectrum). A simple modification, called 'Markovianization', is needed. The essential of this approximation is that the exponential term varies at the nonlinear time scale $\tau_{n l}=\left[\nu\left(k^{2}+p^{2}+q^{2}\right)+\mu_{k p q}\right]^{-1}$, which is much shorter than the time scale governing the evolution of $\sum<u u><u u>$ (on the order of the large-eddy turn-over time). The Eddy-Damped Quasi-Normal Markovian (EDQNM) approximation, therefore, assumes the effect of the time integration only over the exponential part memoral integrals:

$$
\theta_{1}=\int_{0}^{t} \exp \left\{-\left[\nu\left(k^{2}+p^{2}+q^{2}\right)+\mu_{k p q}\right](t-\tau)\right\} f(-p, k, \Omega \tau) d \tau
$$




$$
\begin{aligned}
& \theta_{2}=\int_{0}^{t} \exp \left\{-\left[\nu\left(k^{2}+p^{2}+q^{2}\right)+\mu_{k p q}\right](t-\tau)\right\} f(-q, k, \Omega \tau) d \tau \\
& \theta_{3}=\int_{0}^{t} \exp \left\{-\left[\nu\left(k^{2}+p^{2}+q^{2}\right)+\mu_{k p q}\right](t-\tau)\right\} f(-k, p, \Omega \tau) d \tau, \\
& \theta_{4}=\int_{0}^{t} \exp \left\{-\left[\nu\left(k^{2}+p^{2}+q^{2}\right)+\mu_{k p q}\right](t-\tau)\right\} f(-q, p, \Omega \tau) d \tau \\
& \theta_{5}=\int_{0}^{t} \exp \left\{-\left[\nu\left(k^{2}+p^{2}+q^{2}\right)+\mu_{k p q}\right](t-\tau)\right\} f(-k, q, \Omega \tau) d \tau, \\
& \theta_{6}=\int_{0}^{t} \exp \left\{-\left[\nu\left(k^{2}+p^{2}+q^{2}\right)+\mu_{k p q}\right](t-\tau)\right\} f(-p, q, \Omega \tau) d \tau .
\end{aligned}
$$

Then we obtain

$$
\begin{aligned}
T_{\alpha \beta \gamma}(\mathbf{k}, \mathbf{p}, t)= & i\left(\theta_{1} Q_{\alpha \rho \sigma}(-\mathbf{p}, \mathbf{k}) S_{\beta \rho}(\mathbf{p}, t) S_{\gamma \sigma}(\mathbf{q}, t)+\theta_{2} Q_{\alpha \rho \sigma}(-\mathbf{q}, \mathbf{k}) S_{\beta \sigma}(\mathbf{p}, t) S_{\gamma \rho}(\mathbf{q}, t)+\right. \\
& \theta_{3} Q_{\beta \rho \sigma}(-\mathbf{k}, \mathbf{p}) S_{\alpha \rho}(\mathbf{k}, t) S_{\gamma \sigma}(\mathbf{q}, t)+\theta_{4} Q_{\beta \rho \sigma}(-\mathbf{q}, \mathbf{p}) S_{\alpha \sigma}(\mathbf{k}, t) S_{\gamma \rho}(\mathbf{q}, t)+(\mathrm{A} .6) \\
& \left.\theta_{5} Q_{\gamma \rho \sigma}(-\mathbf{k}, \mathbf{q}) S_{\alpha \rho}(\mathbf{k}, t) S_{\beta \sigma}(\mathbf{p}, t)+\theta_{6} Q_{\gamma \rho \sigma}(-\mathbf{p}, \mathbf{q}) S_{\alpha \sigma}(\mathbf{k}, t) S_{\beta \rho}(\mathbf{p}, t)\right) .
\end{aligned}
$$

Using (A.6) we get expressions for $T_{\beta \rho \sigma}(-k, p, t)$ and $T_{\alpha \rho \sigma}(k, p, t)$ in (A.1). Now we substitute these expressions in (A.1) to obtain

$$
\begin{array}{r}
\left(\frac{\partial}{\partial t}+2 \nu k^{2}\right) S_{\alpha \beta}(k, t)= \\
-\sum_{p} f(p, k, \Omega t) Q_{\alpha \rho \sigma}(p, k)\left(\theta_{1} Q_{\beta \xi \eta}(-p,-k) S_{\rho \xi}(p, t) S_{\sigma \eta}(q, t)+\right. \\
\theta_{2} Q_{\beta \xi \eta}(-q,-k) S_{\rho \eta}(p, t) S_{\sigma \xi}(q, t)+ \\
\theta_{3} Q_{\rho \xi \eta}(k, p) S_{\beta \xi}(-k, t) S_{\sigma \eta}(q, t)+\theta_{4} Q_{\rho \xi \eta}(-q, p) S_{\beta \eta}(-k, t) S_{\sigma \xi}(q, t)+ \\
\left.\theta_{5} Q_{\sigma \xi \eta}(k, q) S_{\beta \xi}(-k, t) S_{\rho \eta}(p, t)+\theta_{6} Q_{\sigma \xi \eta}(-p, q) S_{\beta \eta}(-k, t) S_{\rho \xi}(p, t)\right)- \\
-\sum_{p} f(p,-k, \Omega t) Q_{\beta \rho \sigma}(p,-k)\left(\hat{\theta}_{1} Q_{\alpha \xi \eta}(-p, k) S_{\rho \xi}(p, t) S_{\sigma \eta}(q, t)+\right. \\
\hat{\theta}_{2} Q_{\alpha \xi \eta}(-q, k) S_{\rho \eta}(p, t) S_{\sigma \xi}(q, t)+ \\
\hat{\theta}_{3} Q_{\rho \xi \eta}(-k, p) S_{\alpha \xi}(k, t) S_{\sigma \eta}(q, t)+\hat{\theta}_{4} Q_{\rho \xi \eta}(-q, p) S_{\alpha \eta}(k, t) S_{\sigma \xi}(q, t)+ \\
\left.\hat{\theta}_{5} Q_{\gamma \xi \eta}(-k, q) S_{\alpha \xi}(k, t) S_{\rho \eta}(p, t)+\hat{\theta}_{6} Q_{\sigma \xi \eta}(-p, q) S_{\alpha \eta}(k, t) S_{\rho \xi}(p, t)\right) .
\end{array}
$$

The EDQNM evolution of the second order spectral tensor gives a complete description of the energy transfer and anisotropic structure of rotating turbulence.

Taking trace in (A.7) and combining terms, we obtain an equation for $U(k, t)$.

$$
\left(\frac{\partial}{\partial t}+2 \nu k^{2}\right) U(k, t)=\sum_{p}\left(a^{\prime}(k, p, q, \Omega, t) U(p, t) U(q, t)+\right.
$$


$\left.b^{\prime}(k, p, q, \Omega, t) U(k, t) U(q, t)+c^{\prime}(k, p, q, \Omega, t) U(k, t) U(p, t)\right)$.

In (4.2) it is understood that $\mathbf{k}=\mathbf{p}+\mathbf{q}$. The geometrically determined coefficients $a^{\prime}(k, p, q, \Omega, t)$, $b^{\prime}(k, p, q, \Omega, t)$ and $c^{\prime}(k, p, q, \Omega, t)$ are given by

$$
\begin{aligned}
& a^{\prime}(k, p, q, \Omega, t)=-\frac{1}{4} f(p, k, \Omega t) Q_{\alpha \rho \sigma}(p, k)\left(\theta_{1} Q_{\alpha \xi \eta}(-p,-k) P_{\rho \xi}(\mathbf{p}) P_{\sigma \eta}(\mathbf{q})+\right. \\
& \left.\theta_{2} Q_{\alpha \xi \eta}(-q,-k) P_{\rho \eta}(\mathbf{p}) P_{\sigma \xi}(\mathbf{q})\right)- \\
& \frac{1}{4} f(p,-k, \Omega t) Q_{\alpha \rho \sigma}(p,-k)\left(\hat{\theta}_{1} Q_{\alpha \xi \eta}(-p, k) P_{\rho \xi}(\mathbf{p}) P_{\sigma \eta}(\mathbf{q})+\right. \\
& \left.\hat{\theta}_{2} Q_{\alpha \xi \eta}(-q, k) P_{\rho \eta}(\mathbf{p}) P_{\sigma \xi}(\mathbf{q})\right) \\
& b^{\prime}(k, p, q, \Omega, t)=-\frac{1}{4} f(p, k, \Omega t) Q_{\alpha \rho \sigma}(p, k)\left(\theta_{3} Q_{\rho \xi \eta}(k, p) P_{\alpha \xi}(-\mathbf{k}) P_{\sigma \eta}(\mathbf{q})+\right. \\
& \left.\theta_{4} Q_{\rho \xi \eta}(-q, p) P_{\alpha \eta}(-\mathbf{k}) P_{\sigma \xi}(\mathbf{q})\right)- \\
& -\frac{1}{4} f(p,-k, \Omega t) Q_{\alpha \rho \sigma}(p,-k)\left(\hat{\theta}_{4} Q_{\rho \xi \eta}(-q, p) P_{\alpha \eta}(\mathbf{k}) P_{\sigma \xi}(\mathbf{q})+\hat{\theta}_{3} Q_{\rho \xi \eta}(-k, p) P_{\alpha \xi}(\mathbf{k}) P_{\sigma \eta}(\mathbf{q}) \wedge .10\right) \\
& c^{\prime}(k, p, q, \Omega, t)=-\frac{1}{4} f(p, k, \Omega t) Q_{\alpha \rho \sigma}(p, k)\left(\theta_{5} Q_{\sigma \xi \eta}(k, q) P_{\alpha \xi}(-\mathbf{k}) P_{\rho \eta}(\mathbf{p})+\right. \\
& \left.\theta_{6} Q_{\sigma \xi \eta}(-p, q) P_{\alpha \eta}(-\mathbf{k}) P_{\rho \xi}(\mathbf{p})\right)- \\
& -\frac{1}{4} f(p,-k, \Omega t) Q_{\alpha \rho \sigma}(p,-k)\left(\hat{\theta}_{5} Q_{\gamma \xi \eta}(-k, q) P_{\alpha \xi}(\mathbf{k}) P_{\rho \eta}(\mathbf{p})+\right. \\
& \left.\hat{\theta}_{6} Q_{\sigma \xi \eta}(-p, q) P_{\alpha \eta}(\mathbf{k}) P_{\rho \xi}(\mathbf{p})\right) \text {. }
\end{aligned}
$$

We note that the geometrically determined coefficients $a^{\prime}, c^{\prime}$ and $c^{\prime}$ contain $f$ and $\theta$. The coefficients $\theta$ are given by (A.5). 